\title{
Evidências de validade da Escala de Estresse em Estudantes para universitários brasileiros
}

\author{
Paulo Roberto Taborda de Souza Filho ${ }^{1}$, Sheila Gonçalves Câmara ${ }^{2}$ \\ Universidade Federal de Ciências da Saúde de Porto Alegre-Brasil
}

\begin{abstract}
O estudo apresenta o processo de adaptação e as evidências de validade da Escala de Estresse em Estudantes para a realidade brasileira. Participaram do estudo 585 universitários de instituiçōes brasileiras. Realizaram-se análises fatoriais, tendo sido obtido um modelo de quatro fatores, com alfas satisfatórios (sintomas fisiológicos: $\alpha=.82$, relação professor-aluno: $\alpha=.80$, incapacidade e inferioridade: $\alpha=.77$ e lazer: $\alpha=.75$ ), e adequados índices de ajuste: $x^{2}=282.313(\mathrm{p}=.00) ; x^{2} / \mathrm{gl}=1.73 ; \mathrm{TLI}=.97 ; \mathrm{RMSEA}=.05$ (I.C.=.04-.06); CFI =.97. A escala apresentou correlação positiva significativa $(r=.60, p \leq .01)$, assim como bons índices de sensibilidade (99.2\%) e especificidade (89.6\%) em relação à Escala de Estresse Percebido, utilizada como padrão-ouro. $\mathrm{O}$ instrumento contribui para a ampliação de estudos sobre estresse em estudantes universitários

Palavras-chave: estresse acadêmico, estudante universitário, ensino superior, validação de instrumento, análise fatorial.
\end{abstract}

Evidencias de validez de la Escala de Estrés en Estudiantes para universitarios brasileños El estudio presenta el proceso de adaptación y las evidencias de validez de la Escala de Estrés en Estudiantes para la realidad brasileña. Participaron del estudio 585 universitarios de instituciones brasileñas. Se realizaron análisis factoriales, habiéndose obtenido un modelo de cuatro factores, con alfas satisfactorios (síntomas fisiológicos: $\alpha=.82$, relación profesoralumno: $\alpha=.80$, incapacidad e inferioridad: $\alpha=.77$ y ócio: $\alpha=.75$ ), y adecuados índices de ajuste: $\mathrm{x}^{2}=282.313(p=.00) ; \mathrm{x}^{2} / \mathrm{df}=1.73$; TLI=.97; RMSEA=.05 (I.C.=.04-.06); CFI=.97. La escala presentó correlación positiva significativa $(\mathrm{r}=.60, p \leq .01)$, así como buenos índices de sensibilidad (99.2\%) y especificidad (89.6\%) en relación a la Escala de estrés percebido. El instrumento contribuye a la ampliación de estudios sobre estrés en estudiantes universitarios.

Palabras-clave: estrés acadêmico, estudiantes universitários, educación universitária, validación de instrumentos, análisis factorial.

1 Psicólogo. Mestre em Psicologia e Saúde pela Universidade Federal de Ciências da Saúde de Porto Alegre. Endereço postal: Rua Sarmento Leite, 245, Prédio 1, Sala 208 Porto Alegre, RS, 90050-170. Contato: paulotabordasouza@gmail.com. https://orcid. org/0000-0003-4970-9865

2 Professora adjunta do Departamento do Curso de Psicologia e Programa de Pós-Graduação em Psicologia e Saúde da Universidade Federal de Ciências da Saúde de Porto Alegre. Endereço postal: Rua Sarmento Leite, 245, Prédio 1, Sala 208 - Porto Alegre, RS, 90050-170. Contato: sheila.gcâmara@gmail.com. https://orcid.org/0000-0001-6761-7644 


\section{Evidence of validity for the Student Stress Scale for Brazilian university students}

This study shows the evidence of validity of the Student Stress Scale for Brazilians. It was administered to 585 Brazilian students. Factor Analyses demonstrated the existence of four factors, with high internal consistency indicators (physiological symptoms: $\alpha=.82$, professor-student relationship: $\alpha=.80$, inability and inferiority: $\alpha=.77$ and leisure: $\alpha=.75$ ) and good model fit indexes: $\mathrm{x}^{2}=282.313(p=.00) ; x^{2} / \mathrm{gl}=1.73$; TLI=.97; RMSEA=.05 (I.C. $=.04-$ .06); CFI=.97. Pearson Correlation analysis showed good correlation coefficients for the gold-standard criteria (Perceived Stress Scale; $r=.60, p \leq .01$ ) and good indexes for sensibility $(99.2 \%)$ and specificity (89.6\%). Results indicate that the scale can contribute to new research on academic stress in university students.

Keywords: academic stress, college students, higher education, test validation, factor analysis.

\section{Les preuves de validité de l'échelle de stress des étudiants pour les étudiants universi- taires brésiliens}

L'étude présente le processus d'adaptation et les preuves de validité de l'échelle de stress des élèves pour la réalité brésilienne. Un total de 585 étudiants universitaires d'institutions brésiliennes ont participé à l'étude. L'analyse factorielle a été réalisée en utilisant un modèle à quatre facteurs avec des alphas satisfaisants (symptômes physiologiques: $\alpha=.82$, ratio enseignant-élève: $\alpha=.80$, handicap et infériorité: $\alpha=.77$ et loisirs : $\alpha=.75$ ), et des indices d'ajustement adéquats: $\mathrm{x}^{2}=282.313(p=.00) ; \mathrm{x}^{2} / \mathrm{gl}=1.73$; TLI=.97; RMSEA =.05 (I.C.=.04-.06); CFI=.97. L'échelle avait une corrélation positive significative $(r=.60, p \leq .01)$, ainsi qu'une bonne sensibilité $(99.2 \%)$ et une spécificité (89.6\%) par rapport à l'échelle de stress perçu. L'instrument contribue à l'expansion des études sur le stress chez les étudiants universitaires.

Mots-clés: stress scolaire, étudiant universitaire, l'enseignement supérieur, validation d'instrument, analyse factorielle. 
A universidade pode ser um ambiente bastante estressante para qualquer indivíduo, seja ele docente, discente, técnico ou outro (Assis, Silva, Lopes, Silva \& Santini, 2013). No caso dos estudantes, as pressóes e expectativas exercidas e esperadas podem acabar exacerbando seus níveis de estresse. Os primeiros anos dos acadêmicos na universidade são cruciais no que diz respeito às condiçóes de estresse e mecanismos de enfrentamento. As dificuldades para lidar com a adaptação ao meio acadêmico (aulas, exames, rendimento, ansiedade, competitividade), assim como administrar as pressóes e expectativas de independência e realização acadêmica, estão diretamente relacionadas com os índices de estresse que os indivíduos vivenciam em relação à universidade e ao futuro profissional (Hirsch, Barlem, Tomashcewski-Barlem, Lunardi \& Oliveira, 2011). Essa oscilação emocional intensa que ocorre está sujeita a se constituir em períodos de maior estresse. A literatura aponta que os estudantes apresentam momentos de tensão, tanto em períodos avaliativos quanto durante o processo de ensino e aprendizagem (Panacioni \& Zanini, 2012).

Um estudo de revisão sistemática e meta-análise de intervenções sobre estresse em estudantes universitários demonstrou que os níveis de prevalência de comorbidades do estresse universitário como transtornos de humor e de ansiedade vêm aumentando ao longo das duas últimas décadas (Regehr, Glancy \& Pitts, 2013). Os autores identificaram que, aproximadamente, cerca de $50 \%$ das amostras de estudantes pesquisadas apresentavam alguma sintomatologia significativa de estresse sob o formato de ansiedade ou depressão - com prevalência significativa de ideação suicida (Downs \& Eisenberg, 2012). Isso implica em problemas de relacionamento com outros colegas estudantes, menores níveis de engajamento em atividades relativas à universidade, notas mais baixas e menores taxas de graduação do que quando comparados com estudantes que não apresentam índices de estresse e sintomatologias associadas (Keyes, Eisenberg, Perry, Dube, Kroenke \& Dingra, 2012). 
$\mathrm{Na}$ literatura brasileira, poucos estudos abordam a temática do estresse universitário a partir de uma perspectiva que avalie a prevalência desse fenômeno e de suas contingências de modo mais objetivo. Torquato et. al. (2010) realizaram um estudo de prevalência de sintomas relacionados ao estresse (pelo Genereal Health Questionaire - 12) com uma amostra de 118 estudantes de Fisioterapia do sul do Brasil. Os autores reportaram uma prevalência de $37.76 \%$ de estudantes com sintomatologia psiquiátrica significativa. No entanto, o instrumento utilizado não é específico para estresse, tampouco específico para o âmbito acadêmico. Outro estudo, de Oliveira, Filha, Monteiro, Monteiro e Cunha (2013,) também apresentou prevalência de sintomas de estresse (a partir do Inventário de Sintomas de Stress de Lipp - ISSL) em estudantes de um curso de enfermagem do Norte do Brasil. Um total de $70.7 \%(n=41)$ dos estudantes apresentaram sintomas de estresse, com prevalências para tipos de desgaste somático $(41 \%)$ e psicológico (43.9\%).

A pesquisa de Assis, Silva, Lopes, Silva e Santini (2013) levantou a prevalência de sintomas de estresse pelo ISSL em estudantes de um curso de psicologia do norte do Brasil. Os autores encontraram que $72 \%$ da amostra apresentaram sintomas de estresse em diferentes níveis (alerta, resistência e exaustáo, conforme Lipp, 2002). O estudo de Benavente, Silva, Higashi, Guido e Costa (2014) também apresentou prevalência de estresse em estudantes de enfermagem $(n=151)$ do sudeste do Brasil a partir da utilização de um questionário específico para a população - o Avaliação de Estresse em Estudantes de Enfermagem (AEEE) (Costa \& Polak, 2009). Essa amostra apresentou alto índice de estresse em dois fatores do instrumento (Gerenciamento do Tempo e Formação Profissional). No entanto, esse instrumento somente apresenta validade para estudantes de enfermagem. Um estudo de Faro (2013) também buscou apresentar a prevalência de estresse através da Escala de Percepção de Estresse (Luft, Sanches, Mazo \& Andrade, 2007) em 2157 estudantes de Pós-Graduação do Brasil inteiro. Os resultados demonstraram que a média da amostra ficou acima do ponto médio da escala, prevalecendo médias maiores de estresse em estudantes da regiáo 
Norte do País, do sexo feminino e que nunca tinham trabalhado ou estavam trabalhando no período da pesquisa.

No contexto brasileiro, poucos instrumentos são validados para avaliação do estresse em geral. Entre eles estão: Inventário de Sintomas de Stress de Lipp (ISSL), Inventário de Controle de Estresse de Lipp (Lipp, 2002); Inventário de Sintomas de Stress para Adultos de Lipp (Lima, 2005); Inventário de Estresse em enfermeiros (IEE) (Stacciarini, 2000), Escala de Percepção de Estresse - Versão Reduzida (PSS-10) (Reis, Hino \& Añez, 2010); e o Job Stress Scale (JSS) (Pascoal \& Tamayo, 2004). Tais instrumentos nâo são específicos para o contexto universitário e boa parte deles avalia o estresse a partir de concepçôes quase exclusivas de fatores internos, ignorando as relaçóes com contexto no qual os sintomas se manifestam.

Duas escalas específicas no contexto internacional avaliam fatores estressores dentro do contexto universitário: Student-Life Stress Inventory (SLSI) (Gadzella, 1991) e Nivel de Stress em Estudantes (N.I.S.E.S.T.E) (Alves, 1995). O Student-Life Stress Inventory (SLSI), elaborado por Gadzella (1991), é dividido em sete fatores relacionados a diferentes tipos de estressores: frustraçóes, conflitos, pressóes, mudanças e estresse auto-provocado. No entanto, poucas pesquisas internacionais (Misra \& Castilo, 2004) utilizam o instrumento e sua pesquisa de validação apresenta uma amostra muito pequena, tornando os resultados referentes às suas evidências de validade e fidedignidade pouco consistentes (Gadzella, 1991).

A Escala de Nivel de Stress em Estudantes (N.I.S.E.S.T.E) foi elaborada por Maria Zita Rodrigues Alves em Portugal (Alves, 1995). De acordo com a autora, a escala é dividida em três dimensóes: biológica, psicológica e social. Apesar de seu uso em pesquisas internacionais (Sarmento, 2004; Ramos \& Carvalho, 2007), a escala nunca foi utilizada em pesquisas brasileiras e tampouco é validada para esse contexto.

Percebe-se que há uma deficiência de estudos brasileiros em relaçáo ao estresse em âmbito universitário devido à falta de instrumentos especializados para o contexto nacional. Nesse sentido, o presente estudo buscou, como objetivo principal, adaptar e estudar as evidências de 
validade da Escala de Estresse em Estudantes para o contexto de universitários brasileiros. Os objetivos específicos foram: investigar a estrutura fatorial da escala e identificar padróes de convergência por meio de correlaçốes entre os escores da N.I.S.E.S.T.E com outra medida de estresse (Escala de Estresse Percebida, PSS-10), além de buscar estabelecer pontos de corte, utilizando tal instrumento como padrão-ouro.

\section{Método}

\section{Participantes}

Participaram da pesquisa 785 universitários. $\mathrm{O}$ critério estipulado para o número amostral $(n=490)$ foi estabelecido considerando-se um mínimo de 10 participantes por item da escala N.I.S.E.S.T.E (composta por 29 itens; $n=290$ ) para análise fatorial exploratória, e um mínimo de 200 sujeitos para a análise fatorial confirmatória para modelos complexos (Hair, Anderson, Tatham \& Black, 2010). Dos 785, foram excluídos 200 sujeitos, por não haverem preenchido no mínimo $90 \%$ do questionário, totalizando 585 participantes.

Quanto às características da amostra, participaram estudantes de 19 estados brasileiros, sendo o estado com o maior número de participantes o Rio Grande do Sul (81.9\%). Do total, 79\% eram do sexo feminino. A idade variou de 18 a 62 anos $(M=24.9 ; D P=7.0), 73.7 \%$ estavam na faixa etária dos $18-25$ anos, $18.3 \%$ na faixa dos $26-35$ anos, e $8.6 \%$ acima dos 36 anos. Em termos do tipo de instituição, 74\% dos estudantes estudavam em universidades públicas e, $26 \%$, em privadas. A maioria dos estudantes cursava Ciências Biológicas (71.1\%; $n=416)$, seguido por Ciências Humanas $(20.3 \%$; $n=119)$ e por Ciências Exatas $(8.5 \% ; n=50)$. Quanto à situação ocupacional, $42.9 \%(n=$ 251) estavam trabalhando no momento. Em termos de classificação econômica, $11.54 \%$ pertenciam à classe $\mathrm{A}, 57.7 \%$ à classe $\mathrm{B}, 28.3 \%$ à classe C e $2.4 \%$ à classe D. 


\section{Medição}

Questionário Sociodemográfico e relacionado ao contexto universitário em nivel de graduação: foi elaborado um questionário que aborda características sociodemográficas (estado da federação, sexo, idade, tipo de instituição - pública ou privada, curso, situação ocupacional - trabalha ou não). Também foi empregado o Critério de Classificação Econômica Brasil - CCEB (ABEP, 2013) que propóe uma segmentação econômica, utilizando levantamento de características domiciliares a fim de diferenciar a população em classes econômicas.

Escala de Estresse Percebido - Versão Reduzida (Perceived Stress Scale - PSS-10): Instrumento composto por 10 itens que correspondem à frequência de eventos e acontecimentos recentes ocorridos há pelo menos um mês. Os itens são divididos em dois fatores: percepçóes negativas e percepçóes positivas. Os itens são respondidos em escala Likert, variando de 0 (Nunca) a 4 (Muito frequente). A escala foi traduzida e validada para o contexto brasileiro por Reis et. al. (2010) e já foi utilizada em estudos brasileiros (Moreira \& Furegato, 2013; Sebastião, Santos \& Jesus, 2010, Silva, Keller \& Coelho, 2013). Os valores de consistência interna se apresentaram satisfatórios (acima de .70) para todos os itens através da pesquisa de Reis, Hino e Añez (2010);

Escala de Nivel de Stress em Estudantes (N.I.S.E.S.T.E). Instrumento composto por 29 itens é dividida em três grandes dimensóes relacionadas ao estresse: sintomas biológicos (5 itens), sintomas psicológicos (16 itens) e problemas sociais (8 itens). Há também uma divisão em sete fatores: preocupação com as aulas, incapacidade, dificuldade na relação aluno-professor, aulas/professores e dificuldade de concentração, preocupação com exames, preocupação com os estágios e sentimentos de inferioridade. Os itens são respondidos em escala Likert variando de 1 (Não Concordo) a 5 (Concordo Totalmente). O coeficiente de consistência tanto geral quanto por fator (no modelo de três fatores), apresentados por Alves (1995), foram satisfatórios (acima de .70). O instrumento consegue diferenciar fatores de estresse e relacioná-los ao contexto acadêmico universitário em Portugal. 


\section{Procedimentos de adaptaçáo do Instrumento}

A autora do N.I.S.E.S.T.E, Maria Zita Alves, foi contatada com a finalidade de obter-se a autorização para proceder à adaptação para o contexto brasileiro. Uma vez obtida, contou-se com a colaboração da vice-consulesa de Portugal em Porto Alegre, que auxiliou a na adaptaçáo de termos portugueses para o contexto brasileiro (especificamente em relação a dois itens de caráter mais contextual).

Posteriormente, o instrumento passou por avaliação dos pesquisadores, que realizaram uma primeira adaptação semântica de todos os 29 itens da escala, buscando manter o mesmo sentido e clareza do item original. Essa foi enviada a experts, psicólogos, doutores em Psicologia $(\mathrm{n}=4)$ para que avaliassem a clareza dos itens, simplicidade e validade aparente (credibilidade, precisão e relevância dos itens), conforme modelo proposto por Cassep-Borges, Balbinotti e Teodoro (2010).

As respostas dos experts foram avaliadas pelos pesquisadores, que obtiveram os seguintes resultados: a) nove itens apresentaram dificuldade de clareza e compreensão; b) oito itens foram considerados pouco adequados (mas expressam algum aspecto do construto) ou inadequados (que são irrelevantes e deveriam ser eliminados); c) dois itens tiveram recomendação unânime dos experts no sentido de que deveriam ser eliminados, já que ambos avaliavam aspectos relativos à experiência nos estágios, e não contemplavam, portanto, a totalidade do público-alvo.

Optou-se por acatar as sugestôes acerca dos itens que apresentaram dificuldade de clareza e compreensáo. Estes foram reformulados, obtendo-se uma segunda versáo do questionário, a qual foi aplicada em um grupo de 16 estudantes universitários, acadêmicos do curso de Psicologia, a fim de realizar avaliação semântica, garantindo a adequação do instrumento à amostra. Apesar das reformulaçóes, houve problemas de compreensão em alguns itens (os mesmos apontados pelos juízes) e sugestôes foram apontadas em relação à inadequaçáo de alguns itens (também apontados pelos juízes), assim como a inadequação dos itens relativos às experiências de estágio. 
O questionário sofreu uma nova reformulação semântica. Os nove itens que apresentaram dificuldades de clareza e compreensão foram novamente adaptados e as duas questóes relativas ao estágio foram eliminadas do instrumento, totalizando 27 itens. Foram mantidos itens considerados pouco adequados ou inadequados pelos experts, a fim de manter sentido e distribuição mais próximos à escala original.

\section{Procedimento}

No primeiro semestre de 2013 o estudo foi submetido e aprovado pelo Comitê de Ética em Pesquisa da Universidade Federal de Ciências da Saúde de Porto Alegre (parecer 313.154) e segue as conformidades da resolução 466/2012 do Conselho Nacional de Saúde. Posteriormente à aprovação, os estudantes foram convidados a participar através de contato virtual por e-mail e divulgação pelas redes sociais e por contatos ou grupos relacionados ao público-alvo.

A coleta ocorreu de julho a setembro de 2013. Os instrumentos autoaplicáveis foram disponibilizados através do ambiente virtual SurveyMonkey (ferramenta online para condução de pesquisas e aplicação de questionários). Todos os participantes (com idades igual ou maior que 18 anos) concordaram com o Termo de Consentimento Livre e Esclarecido.

\section{Análise de Dados}

Evidências de validade de construto. A amostra total $(n=585)$ foi dividida, aleatoriamente, pelo programa Statistical Package for Social Sciences $^{\oplus}$ (SPSS) v.17, em dois grupos: 60\% ( $\left.n=341\right)$ para a análise fatorial exploratória de eixos principais e 40\% ( $n=244)$ para a análise fatorial confirmatória. A análise fatorial exploratória foi realizada pelo método de extração por eixos principais, com rotação Oblimin direta, que pressupóe relação entre os fatores, e foi realizada através do programa SPSS. A análise fatorial confirmatória foi realizada utilizando-se o método de estimação de Mínimos Quadrados Ponderados Robustos (Robust Weighted Least Squares), através do programa Mplus ${ }^{\oplus}$ v.6.2. 
Evidências de validade de critério concorrente foram obtidas a partir de análises de Correlação de Pearson, comparando as médias do N.I.S.E.S.T.E com a do PSS-10. Análises de Curvas ROC foram conduzidas com os escores do N.I.S.E.S.T.E e do PSS-10 para obtenção de ponto de corte. A análise da fidedignidade foi realizada utilizando-se os coeficientes alfa de Cronbach.

\section{Resultados}

\section{Análise Fatorial de Exploratória}

A adequaçáo da amostra foi mensurada pelos seguintes critérios: Kaiser-Meyer-Olkin $(K M O=.905)$, teste de esfericidade de Bartlett $\left(x^{2}=3344.679 ; p=.00\right)$ e determinante da matriz de correlaçáo $(a=.00003)$. No total foram extraídos cinco fatores dos 27 itens, a partir da distribuição de seus componentes e de seus autovalores (eigenvalue $>1.000$ ), que explicaram $53.52 \%$ da variância. De acordo com a congruência semântica entre os itens e baseados na estrutura original, os fatores encontrados foram denominados: Sintomas Fisiológicos; Relação Professor-Aluno; Incapacidade e Inferioridade; Relaçóes Interpessoais; e, Lazer.

Foram avaliadas as médias dos itens, que variaram de 1.48 a 3.77 , com desvios-padráo entre 0.95 a 1.33 , o que demonstra a não ocorrência de efeito "chão" (1) ou "teto" (5) em nenhum dos itens analisados. As correlaçóes item-total foram: Sintomas Fisiológicos (entre $r=.57$ e $r=.31$ ), Relação Professor e Aluno (entre $r=.55$ e $r=.37$ ), Incapacidade e Inferioridade (entre $r=.59$ e $r=.37$ ), Relaçóes Interpessoais (entre $r=.29$ e $r=.28$ ), e Lazer (entre $r=.58$ e $r=.46$ ). Para todos os itens a homogeneidade corrigida obteve valores superiores a 30 .

Dentre as médias das subescalas, a dimensão de 'Incapacidade e Inferioridade' obteve o valor mais elevado $(\mathrm{M}=2.57 ; \mathrm{DP}=.81)$, seguido da dimensão de 'Sintomas Fisiológicos' $(\mathrm{M}=2.52 ; \mathrm{DP}=.81)$. Isso quer dizer que altas pontuaçóes nessas dimensóes implicam em maiores índices de Estresse. $\mathrm{O}$ fator que apresentou menor média foi o de 
'Relaçóes Interpessoais' ( $\mathrm{M}=1.58 ; \mathrm{DP}=.79)$. As médias e cargas fatoriais são apresentadas na Tabela 1.

O coeficiente de fidedignidade para a escala geral foi de $\alpha=.88$. Quase todos os itens contribuíram para o aumento da consistência interna do fator ao qual pertencia e da escala toda. No entanto, a supressão do item 1 (do fator 'Sintomas Fisiológicos') aumentaria o alfa em .021. Decidiu-se eliminar o item aumentando a confiabilidade do fator 1 e da escala geral. O fator quatro ('Relaçóes Interpessoais') apresentou um índice de confiabilidade bastante baixo $(\alpha=.47)$. Seus itens também apresentaram as médias mais baixas quando comparados aos demais da escala. $\mathrm{O}$ fator está composto por apenas dois itens, o que não é recomendado para uma estrutura fatorial adequada (Hair et. al., 2010; Laros, 2012). Dessa forma, optou-se por retirar o fator 'Relaçóes Interpessoais' e prosseguir com o modelo de quatro fatores, com 24 itens.

\section{Análise Fatorial Confirmatória}

Obtido o modelo de quatro fatores foi testada a validade de construto através da análise fatorial confirmatória. A análise apresentou índices insatisfatórios de adequação do modelo: $\mathrm{x}^{2}=520.513(\mathrm{p}=.00) ; \mathrm{x}^{2} / \mathrm{gl}=2.11$; TLI=.94; RMSEA= .07 (I.C.=.06-.08); CFI=.94. Foram, então, empregadas análises e modificações post-hoc para promover melhor adequação do modelo ou novos modelos alternativos, como proposto por Lei e Wu (2007). Dois itens apresentaram baixo peso de regressão (itens 2 e 8) e foram eliminados. Os itens 14 e 21 (pertencentes aos fatores 'Sintomas Fisiológicos' e 'Relação Professor-Aluno', respectivamente), apresentaram alta covariância de erro com itens de seu mesmo fator e de outros fatores, justificando sua eliminação. Os índices de modificação apontaram para melhor ajuste do modelo com a realocação do item 13 no fator 'Lazer', com o qual apresenta coerência semântica.

Obteve-se, no final, um modelo de quatro fatores e 20 itens, com correlação entre os erros dos itens 11 e 12 (ambos de Incapacidade e Inferioridade). Esse último modelo (Figura 1) alcançou boa adequação, com todos os índices de ajuste considerados: $x^{2}=282.313(p=.00) ; x^{2} / g l=1.73$; TLI=.97; RMSEA=.05 (I.C.=.04-.06); CFI=.97 (Hair et. al, 2010). 


\section{Tabela 1}

Cargas fatoriais, estatística descritiva e consistência interna dos itens e dimensóes da Escala de Nivel de Estresse nos Estudantes (N.I.S.E.S.T.E) na análise fatorial por eixos principais $(n=341)$

\begin{tabular}{|c|c|c|c|c|c|}
\hline Subescala/Item & $\begin{array}{l}\text { Cargas } \\
\text { fatoriais }\end{array}$ & $\mathrm{M}(\mathrm{DP})$ & $\begin{array}{l}\text { Homoge- } \\
\text { neidade } \\
\text { corrigida }\end{array}$ & Assimetria & $\begin{array}{l}\text { Alfa } \\
\text { sem } \\
\text { item }\end{array}$ \\
\hline Sintomas Fisiológicos $(\alpha=.70)$ & & $2.52(.81)$ & - & .39 & - \\
\hline 16 - Taquicardia & .70 & $2.31(1.30)$ & .60 & .59 & .62 \\
\hline 15 - 'Aperto no estômago' & .70 & $2.93(1.47)$ & .62 & .00 & .60 \\
\hline 18 - Dificuldade para dormir & .67 & $2.65(1.50)$ & .58 & .29 & .62 \\
\hline 17 - Alteraçóes de Peso & .65 & $2.34(1.48)$ & .55 & .59 & .63 \\
\hline 14 - Dificuldade para raciocinar & .64 & $2.23(1.17)$ & .63 & .72 & .62 \\
\hline $\begin{array}{l}4-\text { Nervoso com avaliaçóes } \\
\text { finais }\end{array}$ & .62 & $3.14(1.36)$ & .46 & -.14 & .65 \\
\hline $\begin{array}{l}1 \text { - Mesma calma para prova } \\
\text { que aula }\end{array}$ & .50 & $2.07(1.19)$ & .43 & .99 & .83 \\
\hline \multicolumn{2}{|l|}{ Relaçáo Professor e Aluno $(\alpha=.82)$} & $2.35(.98)$ & - & .52 & - \\
\hline $\begin{array}{l}22 \text { - Distância enter alunos e } \\
\text { professores }\end{array}$ & .79 & $2.30(1.25)$ & .71 & .66 & .75 \\
\hline $\begin{array}{l}23 \text { - Opinião do professor } \\
\text { sempre se sobrepóe }\end{array}$ & .77 & $2.69(1.40)$ & .66 & .27 & .76 \\
\hline $\begin{array}{l}24 \text { - Aborrecido com atitudes de } \\
\text { professores }\end{array}$ & .73 & $2.74(1.37)$ & .63 & .25 & .77 \\
\hline $\begin{array}{l}21 \text { - Inferiorizado ao falar com } \\
\text { professor }\end{array}$ & .59 & $1.82(1.15)$ & .51 & 1.27 & .80 \\
\hline $\begin{array}{l}25 \text { - Relação estritamente } \\
\text { profissional }\end{array}$ & .58 & $2.20(1.24)$ & .52 & .78 & .80 \\
\hline \multicolumn{2}{|l|}{ Incapacidade e Inferioridade $(\alpha=.82)$} & $2.57(0.81)$ & - & .30 & - \\
\hline 11 - Incapaz de êxito nos estudos & .72 & $1.66(1.08)$ & .59 & 1.613 & .80 \\
\hline 9 - Inferior ao estudante ideal & .69 & $2.95(1.39)$ & .63 & .1 & .79 \\
\hline $\begin{array}{l}12 \text { - Incapaz de resolver } \\
\text { problemas acadêmicos }\end{array}$ & .62 & $1.70(1.02)$ & .55 & 1.52 & .80 \\
\hline
\end{tabular}




\begin{tabular}{|c|c|c|c|c|c|}
\hline Subescala/Item & $\begin{array}{l}\text { Cargas } \\
\text { fatoriais }\end{array}$ & $\mathrm{M}(\mathrm{DP})$ & $\begin{array}{l}\text { Homoge- } \\
\text { neidade } \\
\text { corrigida }\end{array}$ & Assimetria & $\begin{array}{l}\text { Alfa } \\
\text { sem } \\
\text { item }\end{array}$ \\
\hline $\begin{array}{l}13 \text { - Culpa por desperdiçar } \\
\text { tempo ao não estar estudando }\end{array}$ & .52 & $2.87(1.42)$ & .46 & .07 & .80 \\
\hline 8 - Irritado com notas de colegas & .50 & $1.85(1.21)$ & .49 & 1.27 & .81 \\
\hline $\begin{array}{l}7 \text { - Dificuldade de concentrar } \\
\text { nos estudos }\end{array}$ & .51 & $3.41(1.31)$ & .45 & -.33 & .81 \\
\hline 3 - Críticas me afetam muito & .51 & $2.81(1.31)$ & .51 & .11 & .80 \\
\hline $\begin{array}{l}5 \text { - Fracasso com resultados em } \\
\text { disciplinas }\end{array}$ & .50 & $3.48(1.31)$ & .53 & -.50 & .80 \\
\hline 2 - Dificuldade de suportar aulas & .42 & $2.38(1.16)$ & .36 & .58 & .82 \\
\hline Relaçóes Interpessoais $(\alpha=.47)$ & & $1.58(.78)$ & - & 1.31 & - \\
\hline 26 - Pessoas discordam de mim & .50 & $1.68(.99)$ & .31 & 1.19 & - \\
\hline $\begin{array}{l}28 \text { - Pouco à vontade com } \\
\text { pessoas do sexo oposto }\end{array}$ & .47 & $1.48(0.95)$ & .31 & 1.99 & - \\
\hline Lazer $(\alpha=.72)$ & & $2.55(.91)$ & - & .23 & - \\
\hline 10 - Estudo não permite lazer & .85 & $2.65(1.34)$ & .62 & .27 & .58 \\
\hline $\begin{array}{l}29 \text { - Culpa por estar na cafeteria } \\
\text { com colegas }\end{array}$ & .56 & $2.06(1.34)$ & .54 & 1.00 & .65 \\
\hline $\begin{array}{l}6 \text { - Sobrecarga de matéria não } \\
\text { permite lazer }\end{array}$ & .59 & $3.77(1.32)$ & .45 & -.88 & .67 \\
\hline $\begin{array}{l}27 \text { - Só participo de lazer } \\
\text { quando me procuram }\end{array}$ & .45 & $1.98(1.24)$ & .44 & 1.08 & .70 \\
\hline
\end{tabular}




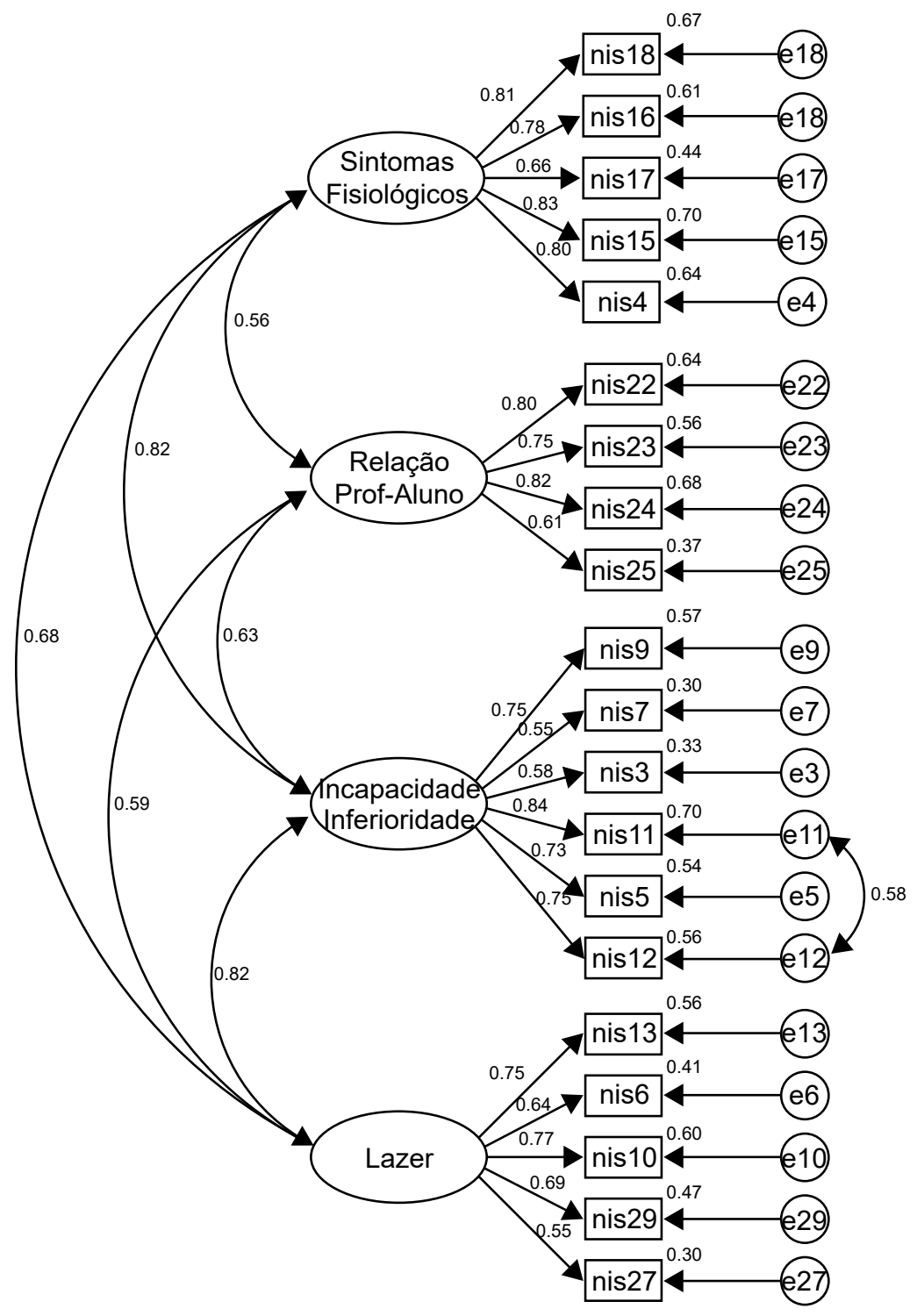

Figura 1. Resultados do modelo fatorial hipotetizado para a Escala de Estresse nos Estudantes (N.I.S.E.S.T.E) ( $\mathrm{n}=244)$. Porto Alegre, RS. 2013. 
A análise da consistência interna do novo modelo revelou índices satisfatórios para a escala geral $(\alpha=.90)$ e seus quatro fatores: Sintomas Fisiológicos $(\alpha=.82)$, Relação Professor e Aluno $(\alpha=.80)$, Incapacidade e Inferioridade $(\alpha=.77)$; e Lazer $(\alpha=.75)$.

Evidências de Validade de Critério. Foram calculadas as médias da N.I.S.E.S.T.E $(M=2.54 ; D P=.79)$ e da PSS-10 $(M=2.19 ; D P=.72)$. As análises de Correlação de Pearson entre as médias dos instrumentos revelaram correlaçóes positivas significativas (Tabela 2), demonstrando que a N.I.S.E.S.T.E possui capacidade de avaliar índices de estresse, discriminando aspectos de estresse geral (PSS-10).

\section{Tabela 2}

Correlaçóes entre as médias dos fatores da Escala de Nivel de Estresse nos Estudantes (N.I.S.E.S.T.E) e médias da Escala de Estresse Percebido Versão Reduzida (PSS-10) (n=585).

\begin{tabular}{lc}
\hline Escala/Fator & PSS-10 \\
\hline Escala Geral (20 itens) & $r=.60(p \leq .01)$ \\
Sintomas Fisiológicos (5 itens) & $r=.51(p \leq .01)$ \\
Relaçáo Professor e Aluno (4 itens) & $r=.36(p \leq .01)$ \\
Incapacidade e Inferioridade (6 itens) & $r=.55(p \leq .01)$ \\
Lazer (5 itens) & $r=.47(p \leq .01)$ \\
\hline
\end{tabular}

Para obtenção de pontos de corte para o escore total da N.I.S.E.S.T.E (relativas à identificação de casos com elevado índice de estresse) foram utilizadas as Análises de Curvas ROC (Receiver Operator Characteristic Curve), utilizando-se como padráo-ouro a PSS-10. O ponto de corte para a PSS-10 foi estabelecido no escore de percentil 75\% (Reis, 2005). O N.I.S.E.S.T.E apresentou bons índices de especificidade e sensibilidade pelos seus valores de pontos de corte. A área embaixo da curva (AUC) também apresentou valores satisfatórios em todas as análises (AUC>.5) (Hajian-Tilaki, 2013) (Tabela 3). 


\section{Tabela 3}

Análise de Curvas ROC entre os escores da Escala de Nivel de Estresse nos Estudantes (N.I.S.E.S.T.E) e escores da Escala de Estresse Percebido (PSS10) (n=585). Porto Alegre, RS, 2013.

\begin{tabular}{lccccc}
\hline $\begin{array}{l}\text { Domínios e } \\
\text { Médias }\end{array}$ & Área & $\begin{array}{c}\text { Intervalo } \\
\text { Confiança } \\
(95 \%)\end{array}$ & $\begin{array}{c}\text { Sensibi- } \\
\text { lidade }\end{array}$ & $\begin{array}{c}\text { Especifi- } \\
\text { cidade }\end{array}$ & $\begin{array}{c}\text { Ponto } \\
\text { Corte }\end{array}$ \\
\hline PSS-10 Geral & .78 & $(.73 ; .82)$ & $99.2 \%$ & $89.6 \%$ & Escore>69.5 \\
$\begin{array}{l}\text { PSS-10 Homens } \\
(\mathrm{n}=123)\end{array}$ & .86 & $(.78 ; .94)$ & $96.2 \%$ & $63.8 \%$ & Escore>64.5 \\
$\begin{array}{l}\text { PSS-10 Mulheres } \\
(\mathrm{n}=462)\end{array}$ & .75 & $(.70 ; .80)$ & $99.0 \%$ & $91.5 \%$ & Escore>69.5 \\
\hline
\end{tabular}

\section{Discussáo}

O presente estudo teve como objetivo adaptar e estudar as evidências de validade da Escala de Nível de Estresse nos Estudantes (N.I.S.E.S.T.E) para o contexto brasileiro. A partir dos resultados psicométricos obtidos, pode-se afirmar que o modelo final se apresenta adequado e satisfatório à avaliação do estresse entre estudantes universitários no território nacional.

Os quatro fatores encontrados (Sintomas Fisiológicos; Incapacidade e Inferioridade; Lazer; e Relação Professor-Aluno) ficaram bem delimitados semanticamente e refletem situaçóes específicas do ambiente acadêmico. Isso revela a capacidade do instrumento adaptado em captar situaçóes estressoras do cotidiano universitário para o estudante (Alves, 1995; Ramos \& Carvalho, 2007). A versão final também apresenta índices satisfatórios de fidedignidade para a escala total e para os fatores independentemente; nesse sentido, pode-se utilizar tanto a escala total quanto os fatores como subescalas para avaliação de variáveis estressoras de maneira específica.

Outra evidência de validade foram os bons valores de ponto de corte obtidos, os quais revelam a capacidade do instrumento para 
discriminar os casos positivos dos negativos relativos aos índices de estresse apresentados pelos estudantes. Isso significa que a escala adaptada é potencialmente útil como instrumento de screening individual, em termos de futuras investigaçóes epidemiológicas acerca da prevalência de estresse nos estudantes (Regehr, Glancy \& Pitts, 2013; Hajian-Tilakim, 2013). Sugere-se que seja utilizado o ponto de corte de 62.5 para a população de estudantes universitários em geral. No entanto, considera-se que é importante avaliar homens e mulheres separadamente, considerando os pontos de corte, por sexo, obtidos na comparação com a PSS-10.

Uma das limitaçóes do estudo diz respeito ao método de coleta de dados. A coleta feita por meio de ferramentas online apresentou número maior de vantagens quando comparada aos meios tradicionais. Entre elas: a facilitação do acesso aos questionários independente de localização geográfica; alcance maior de pessoas em curto espaço de tempo; menor custo com materiais para divulgação e aplicação; dentre outros (Walter, 2013). No entanto, o método de coleta limita os pesquisadores em relação ao controle de algumas variáveis ao longo do estudo. Questóes de seleção e representatividade da amostra (como curso, tipo de universidade e regiáo) tiveram de ser discutidos pelos pesquisadores e relativizadas em detrimento de se obter um número maior de participantes dentro do tempo estimado para a etapa de coleta de dados. Outra limitação encontrada foi relativa à adesão dos sujeitos à pesquisa. $\mathrm{O}$ número de perdas $(n=200)$ é bastante significativo, mas justificável pelo número de instrumentos aplicados (três), o que implica em maior tempo e esforço necessários para serem respondidos, desmotivando os participantes.

É possível, também, fazer algumas considerações acerca das escolhas metodológicas empregadas durante algumas etapas da pesquisa. É importante apontar que diferentes modelos para a N.I.S.E.S.T.E foram explorados e avaliados, a fim de obter o melhor modelo para sua aplicação, conforme Lei e Wu (2007). Como o modelo final apresentou índices satisfatórios, acredita-se que a decisão de avaliar modelos alternativos é bastante adequada e aceitável no contexto da exploração de evidências de validade de instrumentos psicométricos. 
Algumas questôes podem ser levantadas quanto ao modelo original da escala, proposta por Alves (1995). Compreende-se, a partir da investigaçáo em todas as etapas do estudo, que alguns itens se mostraram irrelevantes ao construto de estresse, avaliando, por vezes, outros construtos e/ou que náo seriam adequados ao contexto universitário. Neste sentido, faz-se necessário ressaltar a importância da permissão da autora para que as adaptaçóes e alteraçóes necessárias fossem feitas, visto que as diferenças culturais apresentadas, assim como a atualização conceitual e diferenciaçáo de mensuraçáo do construto, devem ser consideradas durante o processo de adequaçáo de um instrumento para outro contexto (Cassep-Borges et. al., 2010). Optou-se por manter os itens considerados como pouco adequados pelos experts nas análises de eixos principais e fatorial confirmatória. Permaneceram na versão final os itens 22 e 25. Apesar de serem pouco adequados eles apresentam relação com o construto; além disso, seus índices de consistência interna apresentam-se satisfatórios, contribuindo para bons índices de confiabilidade em seus respectivos fatores e na escala total. Na versão final, não constam os itens apontados como inadequados pelos experts na etapa de avaliação de evidências de validade aparente (itens 8,19 , 20, 26 e 28).

A realização desse estudo fez-se necessária devido à falta de instrumentos de mensuração de estresse no âmbito universitário. Há uma escassez de pesquisas com enfoque nessa relação, assim como sáo poucos os estudos brasileiros que abordam aspectos de promoçáo de saúde na universidade e específicos para os estudantes universitários. Acredita-se que, com um instrumento direcionado para essa finalidade, seja possível avaliar o estresse de estudantes nas universidades, de forma a implementar açóes e modelos de promoçáo de saúde nesse contexto. 


\section{Referências}

ABEP (Associação Brasileira de Empresas de Pesquisa). (2013). Critérios de Classificação Econômica Brasil. http://www.abep.org/ novo/FileGenerate ashx?id=297.

Alves, M. Z. (1995). Stress na vida escolar dos estudantes. Tese de Mestrado em Psicologia e Sociologia da Educação. Coimbra, Portugal: Universidade de Coimbra.

Assis, C.L., Silva, A. P., Lopes, M. S., Silva, P. C. \& Santini, T. O. (2013) Sintomas de estresse em concluintes do curso de psicologia de uma faculdade privada do norte do País. Advances in Health Psychology, 21(1), 23-28. https://doi.org/10.15603/2176-1019/ mud.v21n $1 \mathrm{p} 23-28$

Beavers, A. S., Lounsbury, J. W., Richards, J.K., Huck, S. W., Skolits, G. J. \& Esquivel, S. L. (2013) Practical Considerations for Using Exploratory Factor Analysis in Educational Research. Practical Assessment, Research \& Evaluation, 18(6), 1-13. http:// pareonline.net/pdf/v18n6.pdf

Benavente, S. B., Silva, R. M., Higashi, A. B., Guido, L. A. \& Costa, A. L. (2014) Influência de fatores de estresse e características sociodemográficas na qualidade do sono de estudantes de enfermagem. Rev Esc Enferm USP, 48(3), 514-520. http://www.scielo. br/pdf/reeusp/v48n3/pt_0080-6234-reeusp-48-03-514.pdf

Cassepp-Borges, V., Balbinotti, M. \& Teodoro, M. (2010). Tradução e Validação de Conteúdo: Uma Proposta para Adaptação de Instrumentos. Em L. Pasquali (Org.), Instrumentação psicológica: Fundamentos e Práticas (pp. 506-520). Porto Alegre, RS: ArtMed.

Costa, A. L. \& Polak, C. (2009) Construção e validação de instrumento para Avaliação de Estresse em Estudantes de Enfermagem (AEEE). Rev. esc. enferm. USP, 43(spe), 1017-1026. https://doi. org/10.1590/S0080-62342009000500005

Downs, M. F. \& Eisenberg, D. (2012) Help seeking and treatment use among suicidal college students. J Am Coll Health, 60(2), 104-114. https://doi.org/10.1080/07448481.2011.619611 
Faro, A. (2013) Estresse e Estressores na Pós-Graduação: Estudo com Mestrandos e Doutorandos no Brasil. Psicologia: Teoria e Pesquisa, 29(1), 51-60. https://doi.org/10.1590/ S010237722013000100007

Gadzella, B. (1991). Student-Life Stress Inventory. Washington, D.C: ERIC Clearinghouse.

Hair, J. F., Anderson, R. E., Tatham, R. L. \& Black, W. C. (2010). Multivariate Data Analysis ( $7^{\text {th }}$ Edition). Madrid: Pearson-Prentice Hall.

Hajian-Tilaki, K. (2013) Receiver Operating Characteristic (ROC) Curve Analysis for Medical Diagnostic Test Evaluation. Caspian J Intern Med, 4(2), 627-635. https:/www.ncbi.nlm.nih.gov/ pmc/articles/PMC3755824/pdf/cjim-4-627.pdf

Hirsch, C. D., Barlem, E. L., Tomashcewski-Barlem, J. G., Lunardi, V.L. \& Oliveira, A. C., (2014) Preditores do estresse e estratégias de coping utilizadas por estudantes de Enfermagem. Acta Paul Enferm., 28(3), 224-229. https://doi. org/10.1590/1982-0194201500038

Keyes, C. L., Eisenberg, D., Perry, G. S., Dube, S. R., Kroenke, K. \& Dhingra, S. S. (2012) The relationship of level of positive mental health with current mental disorders in predicting suicidal behavior and academic impairment in college students. J Am Coll Health, 60(2), 126-133. https://doi.org/10.1080/07 448481.2011 .608393

Laros, J. A. (2012). O Uso da Análise Fatorial: Algumas Diretrizes para Pesquisadores. Em L. Pasquali (Org.), Análise Fatorial para Pesquisadores (pp. 141-160). Brasília, Brasil:LabPAM.

Lei, P. W. \& Wu, Q. (2007). Introduction to Structural Equation Modeling: Issues and Practical Considerations. Educational Measurement: Issues and Practice, 26(3), 33-43. https://doi. org/10.1111/j.1745-3992.2007.00099.x

Lima, F. V. (2005). Correlação entre variáveis preditoras de estresse e o nivel de estresse. Dissertação de mestrado em Educação Física. Brasília, Brasil:Universidade Católica de Brasília. 
Luft, C. D. B., Sanches, S. O., Mazo, G. Z. \& Andrade, A. (2007). Versão brasileira da escala de estresse percebido: Tradução e validação para idosos. Revista de Saúde Pública, 41(4), 606-615. https://doi.org/10.1590/S0034-89102007000400015

Misra, R., Crist, M. \& Burant, C. (2004). Relationships among life stress, social support, academic stressors, and reactions to stressors of international students in the United States. International Journal of Stress Management, 10(2), 137-157. https://doi.org/10.1037/1072-5245.10.2.137

Moreira, D. \& Furegato, A. (2013). Estresse e depressão entre alunos do último período de dois cursos de enfermagem. Revista Latino-Americana de Enfermagem, 21(spe), 155-162. https:// doi.org/10.1590/S0104-11692013000700020

Oliveira, B. L, Filha, M. de O., Monteiro, C. H., Monteiro, R. V. \& Cunha. C. L. (2012) Estresse entre graduandos de enfermagem de uma universidade pública federal: um estudo epidemiológico. J Manag Prim Health Care, 3(2),72-79. https://doi.org/ 10.14295/jmphc.v3i2.141

Paschoal, T. \& Tamayo, A. (2004). Validação da escala de estresse no trabalho. Estudos de Psicologia, 9(1), 45-55. https://doi. org/10.1590/S1413-294X2004000100006

Ramos, S. I. V. \& Carvalho, A. J. R. (2007). Nivel de stress e estratégias de coping dos estudantes do $1^{\circ}$ ano do ensino universitário de Coimbra. Braga, Portugal: Universidade do Minho. https://estudogeral. sib.uc.pt/bitstream/10316/35175/1/Estudo\%20dos\%20 Niveis\%20de\%20Stress\%20e\%20Estrat\%C3\%A9gias\%20 de\%20Coping\%20nos\%20Alunos\%20de\%20Medicina $\% 20$ da $\% 20$ Faculdade $\% 20 \mathrm{de} \% 20$ Medicina $\% 20 \mathrm{da} \% 20$ Universidade\%20de\%20Co 1 .pdf

Regehr, C., Glancy, D. \& Pitts, A. (2013) Interventions to reduce stress in university students: A review and meta-analysis. Journal of Affective Disorders, 148(1), 1-11. https://doi.org/10.1016/j. jad.2012.11.026 
Reis, R. S. (2005) Comportamentos de Risco à Saúde e Percepção de Estresse em Professores Universitários das Ifes do Sul do Brasil. Florianópolis, Brasil: Universidade Federal de Santa Catarina. https://repositorio.ufsc.br/handle/123456789/102261

Reis, R. S., Hino, A. A. \& Añez, C. R. (2010). Perceived stress scale: reliability and validity study in Brazil. Journal of Health Psychology, 15(1), 107-114. https://doi.org/10.1177/1359105309346343

Sarmento, H. M. B. (2004). Nivel de stress dos alunos do $1^{\circ}$ ano da Faculdade de Ciências do Desporto e Educação Física da Universidade de Coimbra: um estudo descritivo. Coimbra, Portugal:Universidade de Coimbra. Portugal. http://hdl.handle.net/10316/18476

Sebastião, D., Santos, S. \& Jesus, S. (2010). A Influência da cultura/ clima organizacional e da satisfação com o suporte social no estresse percebido. Psychologica, 52(1), 281-300. https://doi.org/ 10.14195/1647-8606_52-1_13

Stacciarini, J. M. \& Tróccoli, B. (2000). Instrumento para mensurar o estresse ocupacional: Inventário de em Enfermeiros (IEEE). Revista Latino-Americana de Enfermagem, 8(6), 40-49. https:// doi.org/10.1590/S0104-11692000000600007

Torquato, J. A., Goulart, A. G., Vicentin, P. \& Correa, U. (2010) Avaliação do estresse em estudantes universitários. InterScience Place, 3(14), 140-154. www.interscienceplace.org/isp/index. $\mathrm{php} / \mathrm{isp} /$ article/view/142

Walter, O. M. (2013). Análise de ferramentas gratuitas para condução de survey online. Produto \& Produção, 14(2), 44-58. https://doi. org/10.22456/1983-8026.22172

Recibido: 18 de abril, 2018

Revisado: 19 de julio, 2019

Aceptado: 31 de julio, 2019 